\title{
REFINANCIAMIENTO COMO UNA PROPUESTA DE SOLUCIÓN \\ PARA PROBLEMAS FINANCIEROS DE LAS EMPRESAS \\ CON UN BANCO PRIVADO
}

\section{REFINANCING AS A PROPOSAL OF SOLUTION FOR FINANCIAL PROBLEMS IN COMPANIES VIA PRIVATE BANK}

Diego Alejandro Wong Cárcamo; Sebastián Alejandro Quiñonez Gutiérrez John Teófilo Santos Medina; Anthony Christian Alcántara Arias

Diego Maldonado Angulo y Juan Carlos Palomino Pinto

Estudiantes de la Universidad Ricardo Palma

Facultad de Ciencias Económicas y Empresariales

Escuela Profesional de Administración de Negocios Globales

Escuela Profesional de Administración y Gerencia

\section{RESUMEN}

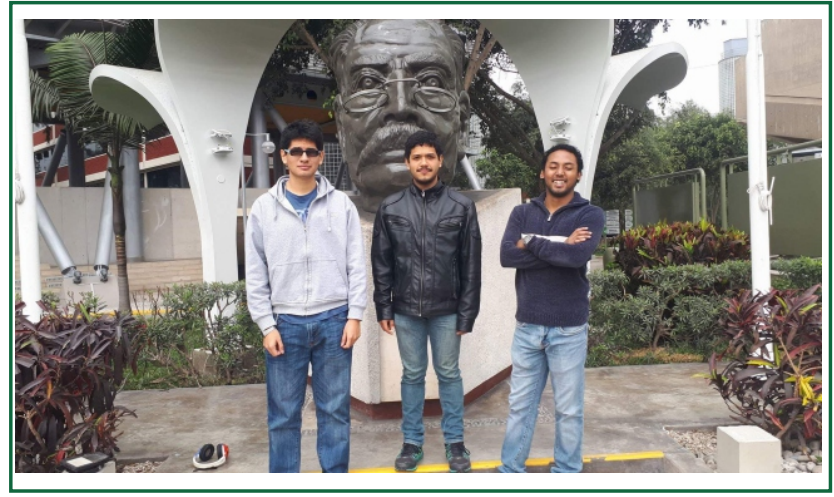

Recepción: 2017-03-27 / Aceptación: 2017-05-08

El objetivo de esta investigación es analizar al refinanciamiento como una propuesta de solución para problemas financieros de empresas con un banco privado en relación a sus deudas y mostrar un caso específico de una empresa privada que solicitó refinanciamiento. Se analizó información sobre el caso de una empresa. Al analizar los problemas financieros que una empresa puede pasar, que básicamente provienen por falta de demanda, se pide un financiamiento, pero cuando este entra en mora se decide solicitar un refinanciamiento mostrándole al banco que el refinancia-miento es una opción idónea para solucionar el problema. Se demuestra que el refinanciamiento es una propuesta de solución para los problemas financieros si es que estos son tomados en cuenta con tiempo y se analiza el costo - beneficio inclusive sin la necesidad de que sea con el mismo banco.

Palabras claves: Financiamiento, refinanciamiento, problema financiero.

\section{ABSTRACT}

The objective of this research is to analyze refinancing as a solution to financial problems in companies with a private bank, in relation to their debts and to show a specific case of a company that has applied for refinancing. Information of a case from a company was made. When analyzing the financial problems that a company may face, which basically come from a low demand, financing is asked for, but when the company cannot pay the debt on the specified time, they will want to apply for refinancing by showing the bank that refinancing is an ideal option for the solution of their problem if they are taken into consideration with time and if cost-benefit is considered even without the necessity of making it with the same bank.

Keywords: Financing, refinancing, financial problem. 


\section{INTRODUCCIÓN}

Se debe entender que para obtener un refinanciamiento se ha debido de realizar previamente un financiamiento reflejado primordialmente como un préstamo. Marquez (2007) señala que la determinación de los efectos del financiamiento se ejecutan de manera que el sistema financiero apoya a las empresas que empiezan a generar utilidades, empleo dentro del sector empresarial $y$, de acuerdo al manejo y a su capacidad, pueden convertirse en empresas altamente rentables.

Para obtener financiamiento existen diversas opciones dentro del mercado, Ixchop (2014) describe las opciones de financiamiento que el empresario puede optar, siendo las más comunes los préstamos con instituciones financieras, los créditos otorgados por los proveedores, los préstamos particulares y el uso del financiamiento interno al reinvertir las utilidades, además los anticipos de clientes representan un financiamiento a corto plazo.

Sin embargo, no todas las empresas a las que se le otorgan financiamiento logran cumplir con el pago, requiriendo de una solución con la entidad prestamista para no afectar tanto su reputación como su situación en el mercado.

Forte y Peña (2009) determinan que el refinanciamiento es uno de los motivos por los cuales las empresas, sean de pequeño o gran tamaño, buscan tener nuevas deudas, además que este refinanciamiento genera una baja en las tasas de interés, a menos que el valor de la empresa crezca.
Lascano (2016) señala que se detectaron engaños en el proceso de refinanciamiento y reestructuración de créditos debido a la inexistencia de procesos la cual provocó negligencias laborales que afectaron la situación financiera de una cooperativa de crédito y ahorro.

En la investigación se tomará en cuenta un banco formal debido a que sus procesos y metodos están mejor regulados.

El objetivo es analizar al refinanciamiento como una propuesta de solución para problemas financieros de las empresas con un banco privado, en relación a sus deudas y mostrar un caso específico de una empresa privada que solicitó refinanciamiento.

\section{MATERIAL Y MÉTODOS}

Se realizaron entrevistas a dos gerentes de bancos privados, se recopiló y analizó información sobre el caso de una empresa privada.

\section{RESULTADOS}

Se considera pertinente dividir la investigación en dos secciones: siendo la primera sobre refinanciamiento y problemas financieros para aplicar al refinanciamiento, y la segunda, un proceso de refinanciación de una empresa privada.

\section{Refinanciamiento}

Los dos principales problemas que dificultan el negocio de las pequeñas y medianas empresas (PYMES) es la falta de demanda y la morosidad 
que sufren a la hora de cobrar los servicios que prestan, esta dificultad obliga a las empresas a requerir de financiamiento de terceros para tener los recursos necesarios para seguir en el mercado. Sin embargo, cuando las empresas siguen en problemas, solventar sus deudas puede volverse complicado y, si la morosidad o demora de pago hacia la entidad del financiamiento se hace permanente, llegará un momento en que acabará cerrando (Tarazona, 2012).

Frente a esta situación las empresas buscan diversas opciones para poder salir del problema. Es importante señalar que el refinanciamiento es la última opción en caso no se pueda solventar las deudas y la empresa se encuentre en muy mal estado financiero.

Se entiende por refinanciamiento a la modificación de las cuotas de la deuda que una empresa o persona tiene por una nueva con un previo acuerdo con la entidad que brindó el financiamiento. En pocas palabras es volver a financiar una deuda.

\section{Problemas financieros para aplicar al refinanciamiento}

Existe una asimetría de información entre los bancos y las empresas lo que conlleva a una dificultad en la entrega de créditos y a su vez genera problemas para las empresas que si obtuvieron el crédito. La asimetría no permite que concuerden los objetivos de la empresa con los del banco demostrándose por las variaciones en los plazos de pago, tasas de interés o capital no suficiente como para poder solventarlo con el tiempo (Lapelle, 2007, citado en Luciano, 2013).
La asimetría se expresa para la empresa que consiguió el crédito como morosidad en los pagos, y este es el problema básico y único por el cual uno puede solicitar refinanciamiento aunque ello no significa que todas las empresas que tienen este problema son aplicables para el refinanciamiento.

Generalmente cada banco posee un propio sistema o método interno por el cuál una empresa o persona natural puede solicitar un refinanciamiento de sus deudas. Estos podrían ser cuotas iniciales que el banco pide como un cobro de servicio, un límite en la cantidad de días que puede estar en mora, un control de puntaje interno que establece por cada empresa que ha obtenido un financiamiento, entre otros; todos ellos varían en distintos bancos.

Tarazona (2012) recomienda que la empresa siga con unos puntos para que pueda obtener solvencia gracias a sus actividades comerciales basadas en un seguimiento estricto para cobros a sus clientes: estimar objetivos dinámicos, que sean continuos y se adecuen a la realidad del cliente (en relación a volúmenes y métodos de pago).

Dichos puntos pueden ser aceptados también para que el banco acepte el riesgo de refinanciar a su deudor, en caso que la solvencia no sea suficiente:

Presentar estados financieros verídicos, si la empresa planea presentarle al banco su propuesta de refinanciamiento necesitará de los datos exactos y actuales de cómo se encuentra la empresa ya que el banco tiene que analizar su riesgo frente a esta situación, separado de la pérdida de reputación de la empresa si trata de engañar a una institución financiera con datos falsos. 
- Ser concreto en los objetivos y demostrar el bien que generará el refinanciamiento de sus deudas. Se logra de la misma manera que uno presenta un proyecto, demostrando cuáles son los objetivos que se han establecido dentro de la empresa junto a lo que se espera llegar rigiéndose de un seguimiento estricto por parte de las dos instituciones que están actuando.

- Establecer contacto permanente con el banco el banco enfrentará periodos de incertidumbre frente al riesgo de refinanciar a un deudor, es por ello que siempre querrá estar al tanto de su situación. Aquí es cuando la información interna de la empresa no debe de cerrar sus puertas al banco, sino todo lo contrario, se debe entregar toda la información que el banco solicite, siendo posible que consulte sobre el flujo de caja, motivos por el cual se ha presentado la incapacidad de pago, el patrimonio neto, estados financieros, estrategias frente a competidores, ventajas competitivas, campañas planeadas, entre otros, para que el banco pueda sustentar la propuesta de refinanciamiento.

La empresa debería tomar en cuenta estos puntos al momento que necesite solicitar un refinanciamiento con la única objeción de que no altere los resultados reales de la situación de la empresa ni sus proyecciones futuras.

Para lograr el segundo objetivo se realizó una entrevista basada en una empresa que solicitó refinanciamiento de sus deudas y se prefirió mantener en el anonimato, por lo cual se conocerá de ahora en adelante a la empresa solicitante del refinanciamiento como "Empresa R". Las entidades financieras participantes fueron el
Banco de Crédito del Perú (BCP) y la financiera Caja Huancayo.

A continuación se presenta el análisis de la empresa que solicitó el refinanciamiento:

En primer lugar, la empresa $\mathrm{R}$ solicitó al Banco de Crédito del Perú (BCP) un préstamo a un plazo de dos años con una tasa de interés que el banco definió en función de distintas variables tales como la situación presente y proyectada de la empresa, el riesgo de incumplimiento de pago, el plazo del crédito, el monto por desembolsar, la competencia, la situación económica del país y del sector económico en el cual la empresa se desenvuelve, entre otros varios aspectos. Pasado un tiempo la empresa $\mathrm{R}$ enfrentó cambios negativos que afectaron significativamente sus niveles de ventas y se estableció que no iban a poder cancelar su deuda respetando el cronograma establecido y menos con una elevada tasa de interés.

Los gerentes de la empresa $\mathrm{R}$ analizaron los efectos que esta situación adversa les causaría y evaluaron qué tanto afectaría la aplicación de la mora sobre su capacidad de pago y concluyeron que era necesario buscar opciones para amortiguar los efectos adversos sobre su capacidad de pago. La solución que encontraron fue el refinanciamiento de la deuda, pero no con el mismo banco. Ellos presentaron una propuesta de refinanciamiento a la entidad financiera Caja Huancayo que consistía básicamente en que la financiera pague el total del préstamo y genere un nuevo pasivo para la empresa $\mathrm{R}$ pero ahora a nombre de la nueva entidad financiera, en otras palabras, consistía en la compra de la deuda por parte de Caja Huancayo. 
La nueva empresa financiera aceptó la propuesta y compró la deuda. Se estableció un nuevo cronograma más holgado con una tasa de interés mucho menor.

Los puntos para evaluar la solicitud y llegar a un posterior acuerdo de refinanciamiento estuvieron basados en la capacidad de generar ingresos o la posibilidad de pagar sus obligaciones, además de presentar a satisfacción de Caja Huancayo documentos como declaraciones y estados financieros presentes y proyectados.

La garantía fue constituida sobre el patrimonio que la empresa $\mathrm{R}$ tenía tanto en bienes muebles como inmuebles. Esta garantía fue un punto esencial que pidió Caja Huancayo debido a que, al ser un caso de refinanciamiento para la empresa $\mathrm{R}$, el riesgo es más alto porque existe el precedente de haber incurrido en incapacidad de pago.

Gracias a ello, la empresa R obtuvo estabilidad financiera la cual permitió cumplir con sus obligaciones y seguir operando en el mercado pese a los cambios negativos producidos en sus niveles de ventas.

\section{CONCLUSIONES}

El refinanciamiento puede llegar a ser una posible solución a los problemas financieros de las empresas que tengan deudas que no puedan pagar con las entidades financieras, que en este caso, los bancos.

Para que el refinanciamiento sea establecido como una solución se tiene que tomar en cuenta los distintos puntos de vista que tiene cada enti- tidad financiera ya que cada uno cuenta con una propia manera de manejar las situaciones con sus clientes.

Estos suelen presentar los estados financieros, estrategias empresariales frente a la competencia, entre otros puntos como por ejemplo lo puede ser un puntaje interno, conocido como "credit scored" o nombres similares dentro de la entidad.

En el caso de la entrevista, hay una gran concordancia en los puntos solicitados por las entidades financieras como la documentación requerida, como si se estuviera generando un nuevo préstamo, aunque si difiere en la manera en cómo se obtuvo ya que la mayoría de autores establecen una relación para solucionarlo con la misma entidad financiera.

Cuando existe un crédito vigente e interviene una tercera figura como prestamista que substituye al anterior y cancela la deuda vigente generando una nueva, la empresa $R$ sabe que se trata de una refinanciación. La nueva empresa financiera que actúa como la tercera figura sabe que ha refinanciado la deuda, pero en cumplimiento de las normas vigentes debe registrar la operación como si fuera un financiamiento nuevo, debido a que no ha substituido ninguna deuda que anteriormente el cliente tuviera registrada con ellos. Adicionalmente, al operar de esa forma no debe constituir provisiones por malas deudas adicionales, y así no reduce las utilidades del periodo.

Por lo tanto, el refinanciamiento puede ser tomado como una propuesta de solución para los problemas financieros sobre todo si es que se actúa con previsión y estos son tomados en cuenta con tiempo. 


\section{REFERENCIAS}

Forte, S. \& Peña, J. I. (2009). Debt refinancing and credit risk. The Spanish Review of Financial Economics, 9 (1), pp. 1-10

Ixchop, D. (2014). Fuentes de Financiamiento de Pequeñas Empresas del Sector Confección de la Ciudad de Matazenango. (Tesis para optar por el grado de licenciatura en administración de empresas). Universidad Rafael Ladívar, Quetzaltenango, Guatemala.

Lascano, G.(2016). Riesgo operativo en el refinanciamiento - reestructuración de créditos y la rentabilidad en la Cooperativa de Ahorro y Crédito Chibuelo. (Tesis para optar por el titulo de ingeniería en contabilidad y auditoría CPA). Universidad Técnica de Ambato, Ambato, Ecuador.
Luciano, M. (2013). Los problemas de financiamiento en las pymes. Universidad Nacional de Cuyo, Facultad de Ciencias Económicas.

Marquez, L. (2007). Efectos del Financiamiento en las Medianas y Pequeñas Empresas. (Tesis para optar por el titulo de profesional de contador público). Universidad San Martín de Porres, Lima, Perú.

Tarazona, A. (2012). Los sistemas de refinanciación de la deuda aplicados por una entidad bancaria en Valencia. Universidad Politécnica de Valencia, Facultad de Administración y Dirección de Empresas.

Correo electrónico para correspondencia diegowongcarcamo@hotmail.com 